\title{
Climate change and control of diarrhoeal diseases in South Africa: Priorities for action
}

\section{Connections between temperature and diarrhoeal disease}

Weather conditions, especially temperature and precipitation, play a critical role in shaping patterns of diarrhoeal diseases. They determine the frequency of outbreaks, and the spatial and seasonal distribution of cases. Not surprisingly, it is anticipated that the burden of diarrhoeal diseases will escalate with climate change, in tandem with gradual increments in mean temperatures, but also during episodic heatwaves. The degree and nature of this escalation will, however, vary with the mix of pathogens in an area, the quality of sanitation services, food hygiene regulations and their enforcement, and the age structure of the population, among other factors. Understanding these patterns can inform the design of measures to prevent and control heat-related diarrhoea. In this editorial, we sum evidence on the heat sensitivity of enteric infections in South Africa (SA) and other parts of sub-Saharan Africa (19 studies), drawing on articles located in a systematic review (methods detailed in Manyuchi et al. ${ }^{[1]}$ ), and consider the implications of these findings for control of diarrhoea in SA in the context of climate change.

Several mechanisms underlie the heat sensitivity of microbes. The reproduction, growth and survival rates of most microbes rise with temperature, within certain limits. Higher ambient temperatures accelerate contamination of food across the whole food chain, from preparation, processing and storage to eventual consumption. Importantly, on hot days people spend more time outdoors, and may eat food that is unrefrigerated and is raw or cold, rather than served cooked and hot. ${ }^{[2]}$ Being outdoors also raises exposure to pathogens carried by birds, and farm and wild animals. Rural areas, per se, may be especially susceptible to heat-related impacts on diarrhoea. ${ }^{[3]}$ Further, dust storms, especially frequent on hot and dry days, may settle dust particles with microbial contaminants onto vegetables and other fresh produce. Importantly, if power blackouts occur during hot weather - as we are currently seeing in SA and Venezuela - the integrity of food chains can be compromised, increasing risks of contamination.

While 15 of the 19 papers identified in the review reported strong connections between heat exposure and cases of diarrhoea, ${ }^{[3-17]} 4$ found negligible or even negative associations. ${ }^{[18-21]}$ This inconsistency illustrates the complexity of pathways between temperature and infectious diseases. Indeed, these correlations are seldom linear and more frequently take an inverted $\mathrm{U}$-shape, where diarrhoea rates rise with temperature, plateau and then decline. Relationships may also be J-shaped, where the incidence of diarrhoea remains stable below a certain temperature threshold, beyond which there is a rapid incline. Moreover, many enteric infections have seasonal patterns: rotavirus cases, for instance, classically occur in winter and campylobacteriosis peaks in spring. ${ }^{[18-20]}$

Seasonal patterns are not solely related to climatic factors, however, and are generally poorly understood. The complexity of transmission dynamics also means that many pathogens are both food- and waterborne, with temperature influencing both modes of transmission. On the plus side, the complexity of these connections means that there are multiple potential points of intervention.

\section{Patterns of heat sensitivity of enteric infections in sub-Saharan Africa}

During periods that are hot and dry, any additional increments in temperature appear to have especially marked impacts on the incidence of diarrhoea in sub-Saharan Africa. ${ }^{[4,8]}$ This is concerning, as periods of hot and dry weather may lengthen and warm further with climate change, and cover ever-larger geographical areas. A study among children aged under 5 in Cape Town, SA, during the hot, dry months of the year, for example, found that a $5^{\circ} \mathrm{C}$ rise in temperature increased cases of diarrhoea by $40 \% 1$ week later. ${ }^{[8]}$ Similar effect sizes were noted in a study in Northern Ghana, at a 2-week lag, ${ }^{[12]}$ and in Limpopo Province, SA. ${ }^{[15]}$ In a study in Mozambique, the incidence of diarrhoea rose by $3.6 \%$ per $1^{\circ} \mathrm{C}$ increase in temperature. ${ }^{[6]}$ Another study, however, using data from demographic health surveys across sub-Saharan Africa, found only weak associations between temperature and cases of diarrhoea in children ${ }^{[21]}$ Additionally, rotavirus occurrence was associated with lower temperatures in several studies, as shown elsewhere. ${ }^{[18-20]}$

Risks of cholera outbreaks are clearly raised by flooding, and interruptions and breakdowns in water and sanitation services, as is presently unfolding in the neighbouring countries of South Africa following Cyclone Idai, but can also be raised during periods of warm weather. ${ }^{[14,16,18]}$ For example, a study in Zambia found that a $1^{\circ} \mathrm{C}$ rise in temperature 6 weeks before the onset of a cholera outbreak explained $5.2 \%$ of the increase in number of cholera cases. ${ }^{[5]}$ The authors of a Tanzanian study reported that an increase of $1^{\circ} \mathrm{C}$ would raise cholera cases by $29 \%$, considerably more than estimates in studies elsewhere. ${ }^{[13]}$

Sea surface temperature appears to predict cholera outbreaks on the subcontinent. ${ }^{[7,9,10,17]}$ The incidence of cholera rose in a linear fashion with sea surface temperature in the KwaZulu-Natal outbreak in $2000 .{ }^{[7]}$ High sea surface temperatures raise risks of heavy precipitation associated with cholera outbreaks, ${ }^{[1]}$ but also enhance phytoplankton growth, leading to higher numbers of copepod zooplankton (small crustaceans commensal to cholera) that feed on these organisms. Cholera infections then occur after people drink untreated water with even a few cholera-containing copepods.

\section{Implications for control of diarrhoea in SA in the era of climate change}

Broadly speaking, there are two approaches to controlling heatrelated diarrhoea. The first entails reducing pathogen concentrations in food chains, water sources and the community in general. ${ }^{[22,23]}$ This approach attenuates the effects of temperature on diarrhoeal infections, potentially counterbalancing the impacts of climate change on case burden. The second approach involves outbreak preparedness, early-warning systems and heat-health action plans, where a specific set of actions are triggered at prespecified temperature thresholds.

Information about the lag time between periods of extreme heat and increases in cases of diarrhoea ( $1-6$ weeks in the studies reviewed) can provide warning about outbreaks and facilitate their early detection, a central pillar of diarrhoea control. In the absence of forecasting and early detection, many unplanned admissions may 
occur, in under-prepared services, and with high morbidity and mortality rates. Lag periods vary considerably, however, contingent on the incubation periods of the pathogen(s) involved, and where and when food contamination occurred. Shorter lags point to heatrelated contamination closer to the time of food consumption, while longer lags indicate exposure at the production and processing stages. Longer lags may also suggest that heat pathways involve an additional step of mechanical vectors, such as flies, which are mostly thermophilic. ${ }^{[4]}$

The recent floods in southern Africa following Cyclone Idai may suggest gaps in preparedness for extreme weather events, at national, regional and international levels. In Mozambique, a cholera outbreak began 2 weeks after the cyclone and oral cholera vaccination was initiated 1 week thereafter, in a large campaign involving almost 1 million doses. ${ }^{[24]}$ It is worth considering whether global stockpiles of the vaccine could have been mobilised earlier, however, based on the expected lags and given the fact that a cholera outbreak occurred in 2018, suggesting favourable conditions for cholera transmission, even pre-cyclone. ${ }^{[25]}$ Experiences with the listeriosis outbreak in SA in 2017/18 indicate that deficiencies in preparedness and outbreak control are not restricted to the neighbouring countries: it took 14 months to identify the outbreak source, and there were substantial gaps in the case histories of affected patients, for example. ${ }^{[2,27]}$

Although heat sensitivity and related causal pathways vary considerably between pathogens and settings, heat-health action plans can set out graded actions, where sets of preventive and control measures are implemented at different temperature thresholds. These actions include rapidly sharing information with health professionals and the public on levels of risk; promoting food safety and safe water use; making provision for additional case burdens in health services; shifting from surveillance of all-cause diarrhoea to making specific pathogen diagnoses; and specific measures such as cholera vaccination. ${ }^{[23]}$

\section{Conclusions}

Average temperatures in SA are anticipated to escalate by $2-4^{\circ} \mathrm{C}$ in the forthcoming decades ${ }^{[28]}$ Risks of cholera and other outbreaks clearly increase during disasters, but now also need to be recognised as important phenomena during heatwaves and the increasingly high temperatures that characterise climate change. The overall burden of diarrhoeal diseases may therefore increase considerably, especially among lower socioeconomic groups and children. Understanding these impacts can help delineate high- and low-risk periods, provide insights into the epidemiology of enteric diseases in the country, identify remedial actions, and help prioritise diarrhoeal diseases in heat-health action plans. Developing and adequately resourcing such plans, which encompass disaster preparedness, should form a key part of SA's climate change response in the coming years.

Acknowledgements. A grant from the Humanities and Social Sciences Youth Foundation of the Ministry of Education of China (13YJCZH167) supported the work of Chongying Wang on this project.

Melanie Boeckmann, ${ }^{1}$ Thomas Roux, ${ }^{2}$ Matthew Robinson, ${ }^{3}$ Ashtyn Areal ${ }^{4}$ Dilara Durusu, ${ }^{4}$ Bianca Wernecke, ${ }^{5}$ Albert Manyuchi, ${ }^{6}$ Minh Duc Pham, ${ }^{7}$ Chongying Wang, ${ }^{8}$ Robyn Hetem, ${ }^{9}$ Lois Harden, ${ }^{10}$ Emily Vargas, ${ }^{11}$ Caradee Y Wright, ${ }^{12}$ Barend F N Erasmus, ${ }^{6}$ Helen Rees, ${ }^{13}$ Coleen Vogel,${ }^{6}$ Shanshan Wang, ${ }^{14}$ Vivian Black, ${ }^{15}$ Mellisa Mabhikwa, ${ }^{13}$ Matthew F Chersich; ${ }^{13}$ Climate Change and Heat-Health Study Group
${ }^{1}$ Department of Environment and Health, School of Public Health Bielefeld University, Germany

${ }^{2}$ School of Public Health, Physiotherapy and Sports Science, University College Dublin, Ireland

${ }^{3}$ Public Health Department, Health Services Executive, Ireland

${ }^{4}$ Maastricht University, Netherlands

${ }^{5}$ Environment and Health Unit, South African Medical Research Council, Johannesburg, South Africa; and Environmental Health Department, Faculty of Health Sciences, University of Johannesburg, South Africa

${ }^{6}$ Global Change Institute, University of the Witwatersrand, Johannesburg, South Africa

${ }^{7}$ Burnet Institute, Melbourne, Australia; and Department of Epidemiology and Preventive Medicine, Faculty of Medicine Nursing and Health Science, Monash University, Melbourne, Australia

${ }^{8}$ Center for Behavioural Sciences, School of Medicine, Nankai University, Tianjin, China

${ }^{9}$ School of Animal, Plant and Environmental Sciences, Faculty of Science, University of the Witwatersrand, Johannesburg, South Africa

${ }^{10}$ Brain Function Research Group, School of Physiology, Faculty of Health Sciences, University of the Witwatersrand, Johannesburg, South Africa

${ }^{11}$ EVidence Knowledge Brokering in Public Health SAS, Bogotá, DC, Colombia

${ }^{12}$ Environment and Health Research Unit, South African Medical Research Council, Pretoria, South Africa; and Department of Geography, Geoinformatics and Meteorology, Faculty of Natural and Agricultural Sciences, University of Pretoria, South Africa

${ }^{13}$ Wits Reproductive Health and HIV Institute, Faculty of Health Sciences, University of the Witwatersrand, Johannesburg, South Africa

${ }^{14}$ Zhou En Lai School of Government, Nankai University, China

${ }^{15}$ Department of Clinical Microbiology and Infectious Diseases, Faculty of Health Sciences, University of the Witwatersrand, Johannesburg, South Africa

Corresponding author: M F Chersich (mchersich@wrhi.ac.za)

1. Manyuchi AE, Areal A, Wernecke $\mathrm{B}$, et al. The impacts of heat on health and effectiveness of interventions to reduce these impacts: A systematic review in the era of climate change. PROSPERO 2018 CRD42018118113. Http//www_crd york.ac_uk/PROSPERO/display_record. hp??ID=CRD42018118113 (accessed 30 April 2018).

2. Kapwata T, Mathee A, le Roux WJ, Wright CY, Diarrhoeal disease in relation to possible household 2. Kapwata T, Mathee A, le Roux WJ, Wright CY. Diarrhoeal disease in relation to possible household
risk factors in South African villages. Int J Environ Res Public Health 2018;15(8):1665. https://doi. org $/ 10.3390 /$ ijerph 15081665

3. Thiam S, Diene AN, Sy I, et al. Association between childhood diarrhoeal incidence and climatic factors in urban and rural settings in the health district of Mbour, Senegal. Int J Environ Res Public Health 2017;14(9):16. https://doi.org/10.3390/ijerph14091049

4. Alexander KA, Carzolio M, Goodin D, Vance E. Climate change is likely to worsen the public health threat of diarrheal disease in Botswana. Int J Environ Res Public Health 2013;10(4):1202-1230. https:// doi.org/10.3390/ijerph10041202

5. Fernandez MAL, Bauernfeind A, Jimenez JD, Gil CL, El O, Guibert DH. Influence of temperature and rainfall on the evolution of cholera epidemics in Lusaka, Zambia, 2003 - 2006: Analysis of a time series. Trans R Soc Trop Med Hyg 2009;103(2):137-143. https://doi.org/10.1016/j.trstmh 2008.07 .017 . Horn LM, Hajat A, Sheppard L, et al. Association between precipitation and diarrheal disease in Mozambique. Int J Environ Res Public Health 2018;15(4):709. https://doi.org/10.3390/ijerph15040709 Mendelsohn J, Dawson T. Climate and cholera in KwaZulu-Natal, South Africa: The role of environmental factors and implications for epidemic prepared j.ijheh.2006.12.002

8. Musengimana G, Mukinda FK, Machekano R, Mahomed H. Temperature variability and occurrence of diarrhoea in children under five-years-old in Cape Town metropolitan sub-districts. Int J Environ Res Public Health 2016;13(9):12. https://doi.org/10.3390/ijerph13090859

9. Reyburn R, Kim DR, Emch M, Khatib A, von Seidlein L, Ali M. Climate variability and the outbreaks of cholera in Zanzibar, East Africa: A time series analysis. Am J Trop Med Hyg 2011;84(6):862-869. https://doi.org/10.4269/ajtmh.2011.10-0277

10. De Magny GC, Cazelles B, Guégan JF. Cholera threat to humans in Ghana is influenced by both global and regional climatic variability. EcoHealth 2006;3(4):223-231. https://doi.org/10.1007/s10393-006$0061-5$

11. De Magny GC, Thiaw W, Kumar V, et al. Cholera outbreak in Senegal in 2005: Was climate a factor? PLoS One 2012.7(8):e44577. https://doi.org/10.1371/journal pone.0044577

2. Seidu R Stenström TA, Löfmon A comparative cohort study of the effect of rinfall and temperature Seidu R, Stenstrom TA, Lofman O. A comparative cohort study of the effect of rainfall and temperature Water Climate Change 2013;4(2):90-102. https://doi.org/10.2166/wcc.2013.032

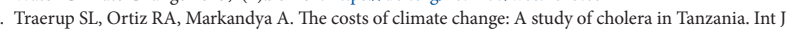
Environ Res Public Health 2011;8(12):4386-4405. https://doi.org/10.3390/ijerph8124386 
14. Munyuli MT, Kavuvu JM, Mulinganya G, Bwinja GM. The potential financial costs of climate change on health of urban and rural citizens: A case study of Vibrio cholerae infections at Bukavu Town, South on health of urban and rural citizens: A case study of Vibrio cholerae infections at Bukavu Town, South
Kivu Province, eastern of Democratic Republic of Congo. Iran J Public Health 2013;42(7):707-725.

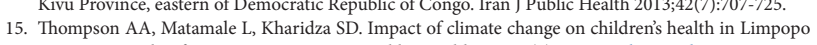
Thompson AA, Matamale L, Kharidza SD. Impact of climate change on children's health in Limpopo
Province, South Africa. Int J Environ Res Public Health 2012;9(3):831-854. https://doi.org/10.3390/ ijerph 9030831

16. Utsalo SJ, Eko FO, Antia-Obong EO. Features of cholera and Vibrio parahaemolyticus diarrhoea endemicity in Calabar, Nigeria. Eur J Epidemiol 1992;8(6):856-860. https://doi.org/10.1007/ BF00145332

17. De Magny GC, Guégan JF, Petit M, Cazelles B. Regional-scale climate-variability synchrony of cholera epidemics in West Africa. BMC Infect Dis 2007;7:9. https://doi.org/10.1186/1471-2334-7-20

18. Gomwalk NE, Umoh UJ, Gosham LT, Ahmad AA. Influence of climatic factors on rotavirus infection among children with acute gastroenteritis in Zaria, northern Nigeria. J Trop Pediatr 1993;39(5):293297. https://doi.org/10.1093/tropej/39.5.293

19. Haffejee IE, Moosa A. Rotavirus studies in Indian (Asian) South African infants with acute gastroenteritis: I. Microbiological and epidemiological aspects. Ann Trop Paediatr 1990;10(2):165-172. https://doi.org/10.1080/02724936.1990.11747425

20. Ouedraogo N, Ngangas SMT, Bonkoungou IJO, et al. Temporal distribution of gastroenteritis viruses in Ouagadougou, Burkina Faso: Seasonality of rotavirus. BMC Public Health 2017;17:8. https://doi. org/10.1186/s12889-017-4161-7

21. Bandyopadhyaya S, Kanjib S, Wanga L. The impact of rainfall and temperature variation on diarrheal prevalence in sub-Saharan Africa. Appl Geogr 2012;33(1):63-72. https://doi.org/10.1016/j. apgeog.2011.07.017
22. Lake IR, Gillespie IA, Bentham G, et al. A re-evaluation of the impact of temperature and climate change on foodborne illness. Epidemiol Infect 2009;137(11):1538-1547. https://doi.org/10.1017/ S0950268809002477

23. Lake IR, Barker GC. Climate Change, Foodborne pathogens and illness in higher-income countries. Curr Environ Health Rep 2018;5(1):187-196. https:///doi.org/10.1007/s40572-018-0189-9

24. McKirdy E, CNN. WHO steps up response as Cyclone Idai cholera cases skyrocket. 2 April 2019. McKirdy E, CNN. WHO steps up response as Cyclone Idai cholera cases skyrocket. 2 April 2019 .
https://edition.cnn.com/2019/04/02/africa/cyclone-idai-who-cholera-intl/index.html (accessed 30 April https:// 2019 ).

25. World Health Organization. Emergencies preparedness, response: Disease outbreak news. Cholera Mozambique. 2018. https://www.who.int/csr/don/19-february-2018-cholera-mozambique/en/ (accessed 30 April 2019).

26. World Health Organization. Emergencies preparedness, response. Disease outbreak news. Listeriosis South Africa. 2018. https://www.who.int/csr/don/28-march-2018-listeriosis-south-africa/en/ (accessed 30 April 2019).

27. Chersich MF, Scorgie F, Rees H, Wright CY. How climate change can fuel listeriosis outbreaks in South Africa. S Afr Med J 201 8;108(6):453-454. https://doi.org/10.7196/SAMJ.2018.v108i6.13274

28. Engelbrecht F, Adegoke J, Bopape MJ, et al. Projections of rapidly rising surface temperatures over Africa Africa under low

S Afr Med J 2019;109(6):359-361. DOI:10.7196/SAMJ.2019.v109i6.14075 